\title{
MODELING OF SPACE-CHARGE PHENOMENA IN MULTI-TURN TRACKING
}

\author{
R.A. Baartman, M. D'yachkov, and F.W. Jones \\ TRIUMF, 4004 Wesbrook Mall, Vancouver, V6T 2A3, Canada
}

\begin{abstract}
Although many space-charge simulations have been developed for linacs, beamlines, and idealized rings, there are relatively few simulation codes applicable to multiturn tracking with space charge in a realistic synchrotron or accumulator/storage ring lattice. Recently the program ACCS IM, used for accumulator-ring injection studies and other applications, has been upgraded with improved transverse space charge models: one using a conventional multiple-Fourier-transform (MFT) method and another using a new hybrid fast-multipole (HFM) algorithm. After an overview of their implementation in the ACCS IM code, we present tests and observations of how well various spacecharge phenomena can be reproduced by the HFM models.
\end{abstract}

\section{INTRODUCTION}

In the class of tracking codes devoted to multi-particle simulations in synchrotrons or accumulator/storage rings, there are several that incorporate space-charge effects, but in the past the use and development of these codes have been curtailed by the intensive computational resources required by the space-charge algorithms, which for most applications have escalated the codes to the supercomputer realm.

Recently, the need for such simulations has increased due to a new generation of intense-beam proton machines that are under study, in the proposal stage, or under construction. These machines must reach new intensity frontiers and be able to operate with extremely low losses, which sets a new standard of accuracy and generality for the simulation codes. Fortunately, at the same time the available computing resources, on the desktop or on modestly-priced compute servers, have increased dramatically and have spurred a new effort to refine and extend the simulation codes.

We have utilized one such code, ACCSIM, to explore some space-charge phenomena with various simulated particle ensembles in an idealized continuous-focusing model. Application to a real machine, the CERN PSB, is described in another paper[1].

\section{TRANSVERSE SPACE CHARGE IN ACCSIM}

The code ACCS IM[2] has been used to study injection and accumulation scenarios for a number of different proton rings, both existing and proposed, as well as being applied to miscellaneous simulation tasks such as rf capture, esti- mation of diagnostic signals, and collimation systems. The numerous simulation features are all built on top of a basic $3 \mathrm{D}$ (6 phase-space variables) tracking engine using transfer matrices to represent the magnet lattice.

Recently, the first self-consistent treatment [1] of transverse space-charge was added to the code, using the standard field-solve/particle-push integration method to incorporate space-charge forces in the tracking. In addition to a conventional FFT-based field solver, based on routines contributed by ACCSIM users at BNL and ORNL [3], a new Hybrid Fast-Multipole solver has been developed. It combines a Particle-In-Cell grid with a Fast-Multipole field evaluation, and can offer advantages in speed and/or accuracy in cases such as few-turn betatron stacking or multiturn injection with halo formation. A comparison of the computational efficiencies of the two methods can be found in Reference [4].

\section{TESTS}

In 2D, the only exactly solvable distribution is the Kapchinsky-Vladimirsky distribution. For simplicity, we have run cases approximating 'smooth-focusing': one thin stigmatic lens every $\sim 10^{\circ}$ of betatron phase advance, and one space charge calculation at every lens. The rms sizes in $x$ and $y$ are calculated and an FFT performed on these. Even with no seeding and matched initial distributions, there is enough numerical noise to give clean spectra. See Fig. 1. Runs were made for equal $x$ - and $y$-emittances and for fixed bare tune in $x\left(\nu_{0 x}=5.817\right)$ and varying tune in $y$ $\left(\nu_{0 y}\right)$. The tune shift in all cases was $\Delta \nu_{x}=0.175$. Fig. 2 shows these tracking results as points. The curves on this figure are the theoretical envelope eigenmode frequencies given by the well-known formula[6, e.g.]

$$
\begin{aligned}
\nu^{2}= & 2 \nu_{0 x}^{2}+2 \nu_{0 y}^{2}-5 \nu_{0 x} \Delta \nu_{x}+ \\
& \pm \sqrt{\left(2 \nu_{0 x}^{2}-2 \nu_{0 y}^{2}\right)^{2}+\left(\nu_{0 x} \Delta \nu_{x}\right)^{2}} .
\end{aligned}
$$

The agreement between tracking and theory is strikingly good. For comparison, we also show twice the bare tunes and twice the incoherent tunes.

In 1971 Sacherer[5] proved that the KV envelope equations apply to any distribution, provided that the beam size is replaced by twice the rms size and the emittance by 4 times the rms emittance. Although the resulting equations are still correct, they are no longer closed, since rms emittance is not a conserved quantity in the presence of nonlinear forces. In practice, however, this turns out to only cause difficulty if one wishes to study the evolution of an 


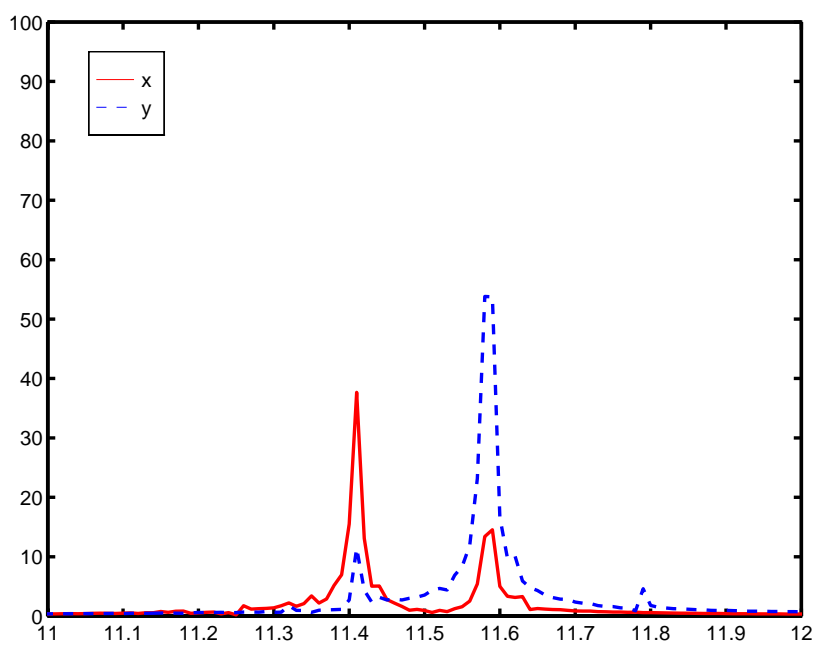

Figure 1: FFT spectra of rms envelopes for $\mathrm{KV}$ case. 10,000 macro-particles. The bare tunes are 5.817 in $x$ and 5.894 in $y$. The tune shift is 0.175 .

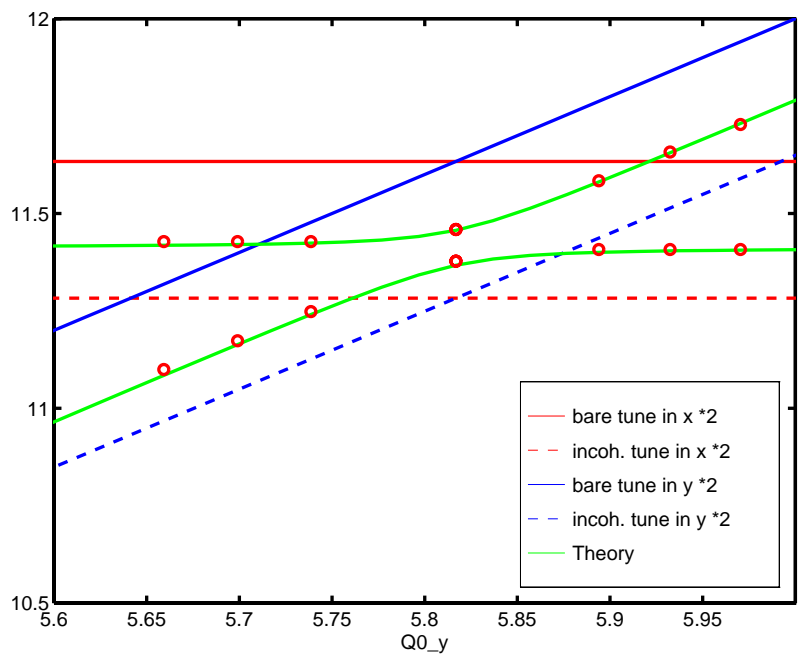

Figure 2: Envelope mode frequencies found from tracking KV beams with 10,000 macro-particles (points) compared with theory (curves) and twice the bare tunes (solid) and twice the incoherent tunes (dashed) plotted against bare tune in $y$. Bare tune in $x$ is 5.817 .

unstable distribution. This was tested in ACCS IM by simulating a gaussian beam. See Fig. 3; the spectra are not as clean as in the KV case, but the modes are still identifiable. These are the points denoted as '+'-symbols in Fig. 4. Again, there is good agreement with the theoretical tunes given by eqn. 1 .

Another test is against the known fact that the KV distribution is stationary. This artificial distribution is an infinitesimally thick shell in 4D phase space. Knowing the matched distribution, each particle can be assigned an 'action' ( $J_{x}$ in $x$ and $J_{y}$ in $y$ ), which is in fact the emittance traced out in phase space as the particle undergoes betatron

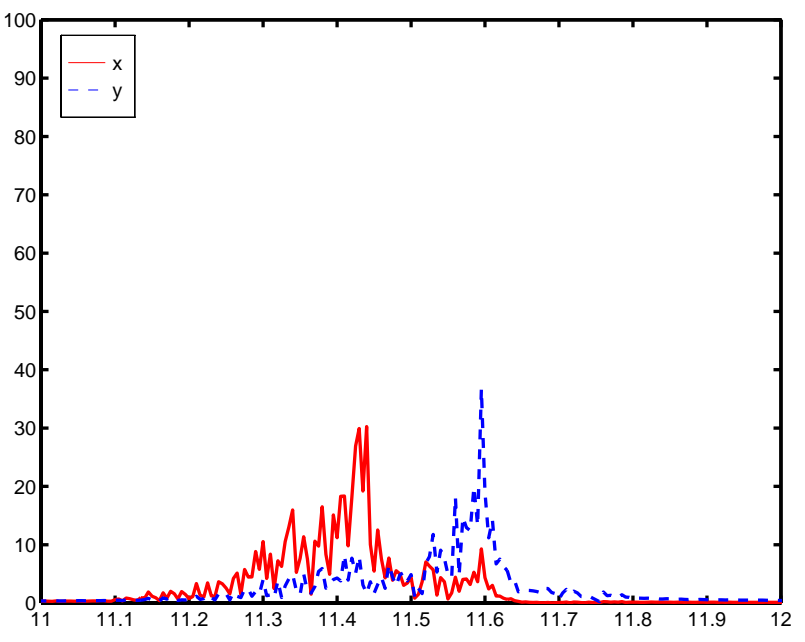

Figure 3: FFT spectra of rms envelopes for gaussian case with 10,000 macro-particles. The bare tunes are 5.817 in $x$ and 5.894 in $y$. The rms emittance is the same as the rms emittance that was used for the KV case. This results in a peak incoherent tune shift of 0.35 .

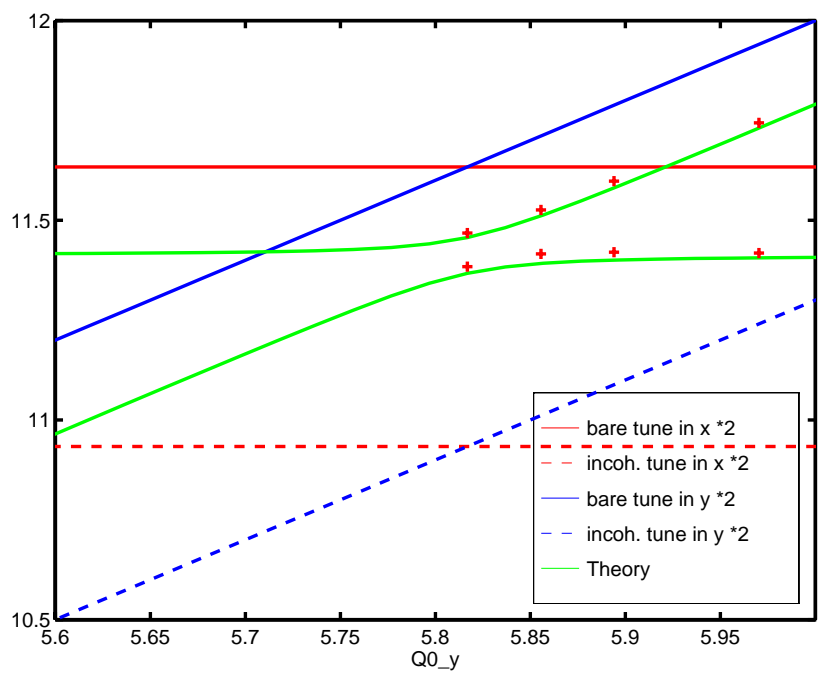

Figure 4: Envelope mode frequencies found from tracking gaussian beams with 10,000 macro-particles (points) compared with theory (curves) and twice the bare tunes (solid) and twice the incoherent tunes (dashed) plotted against bare tune in $y$. Bare tune in $x$ is 5.817 .

oscillations. All the particles in the KV distribution therefore lie on the line $J_{x}+J_{y}=$ constant. In fact, the finite resolution in tracking a finite number of macro-particles results in deviations from this rule so that eventually there is a distribution in $J_{x}+J_{y}$. See Fig. 5. The width of this distribution is plotted in Fig. 6 as a function of turn number. We see that the extra emittance grows quickly to a level which varies as the square root of the number of macro-particles, and thereafter grows only very slowly. This is an 'acid'-test for the tracking method, since the KV case has hard edges 
that require very high resolution to maintain. Tracking with similar parameters and gaussian distributions, for example, yields no detectable diffusion.
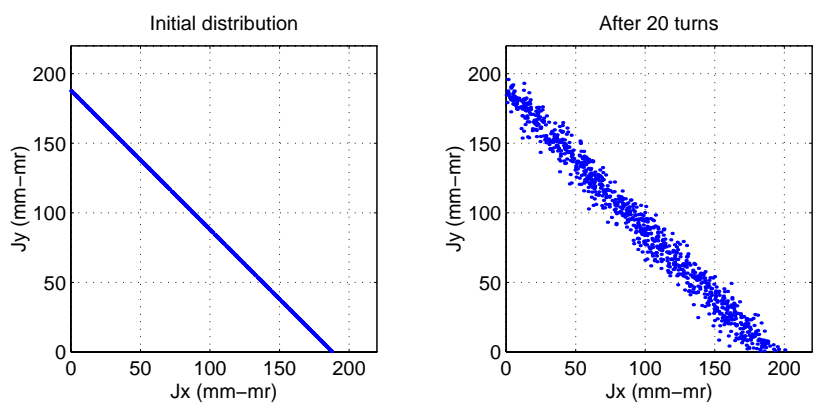

Figure 5: Numerical diffusion of an initial KV beam. 40,000 particles, of which only every $40^{\text {th }}$ has been plotted.

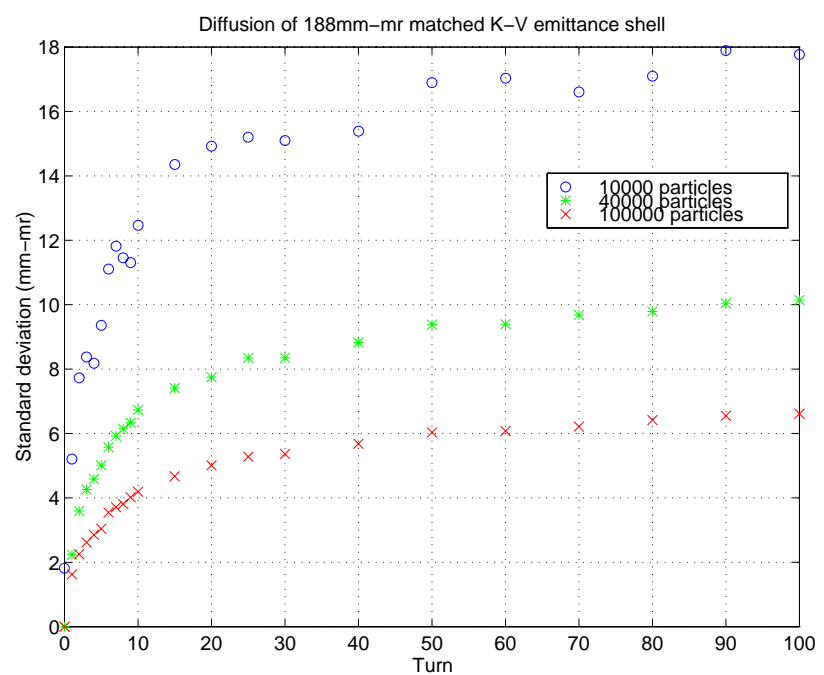

Figure 6: Numerical diffusion of an initial $\mathrm{KV}$ beam of emittance $188 \pi \mathrm{mm}$-mrad, for three different numbers of macro-particles.

\section{CONCLUSION}

Tracking with the new hybrid fast-multipole algorithm shows good agreement with analytical results.

\section{REFERENCES}

[1] F.W. Jones and H.Schönauer, "New Space-Charge Methods in ACCS IM and Their Application to Injection in the CERN PS Booster," these proceedings.

[2] F.W. Jones, "Developments in the ACCSIM Multi-particle Tracking and Simulation Code," Proc. 1997 Particle Accelerator Conference, Vancouver, 1997, p. 2597.

[3] J.A. Holmes et al., "A Particle-In-Cell Model for SpaceCharge Dynamics in Rings," Proc. European Particle Accelerator Conference, Stockholm, 1998.
[4] F.W. Jones, "A Hybrid Fast-Multipole Technique for SpaceCharge Tracking With Halos," Workshop on Space Charge Physics in High Intensity Hadron Rings, Shelter Island NY, May 1998, AIP Conf. Proc. 448 p. 359.

[5] F. Sacherer, RMS Envelope Equations with Space Charge IEEE Trans. Nucl. Sci. NS-18 (PAC 1971) p. 1105.

[6] R. Baartman, "Betatron Resonances with Space Charge," Workshop on Space Charge Physics in High Intensity Hadron Rings, Shelter Island NY, May 1998, AIP Conf. Proc. 448 p. 56.

[7] ACCSIM home page,

http://www.triumf.ca/compserv/accsim.html. 